\title{
Analysis of Cooperative and Competitive Spectrum Sharing for \\ Heterogeneous Networks Based on Differential Dynamics Model
}

\author{
Xiaorong Zhu, Mehrdad Dianati, and Hongbo Zhu
}

\begin{abstract}
The heterogeneous networks belonging to different service providers (SPs) form a coalition system for maximizing the profit, where they may either compete or cooperate with each other. In this paper, we introduce Lokta-Volterra model, a differntial dynamics model, to build the competitive and cooperative mechanisms of heterogeneous networks. It considers the natural growth rate of the network itself and competitive and cooperative effects among networks. Then, according to ordinary differential principle, the stability of the proposed model and its equilibrium points are analyzed. And system performances are evaluated by Vensim which is used for developing, analyzing, and packaging dynamic feedback models. Analysis and simulation results show that the natural growth rate of the network cannot increase its profit but effective cooperative mechanism among heterogeneous networks can increase the profit of each network.
\end{abstract}

Index Terms - Heterogeneous networks, cooperative and competitive, spectrum sharing, Lokta-Volterra model.

\section{I.Introduction}

With the development of wireless communication technology, a variety heterogeneous communication technologies provide the users with ubiquitous access to the data networks. In heterogeneous wireless networks, user terminal with multi-network interface is capable of accessing different networks and choose the network with the most suitable quality of service (QoS) with the least service costs. In such an environment, all the service providers (SPs) compete to maximize their revenue by attracting more users. As a result, severe competition may result in lower product prices and may shrink total profits of SPs in turn, which may not be desirable for SPs. In that case, SPs may opt to cooperate instead of competition. To this end, the cooperation among heterogeneous networks has become an important research topic [1]-[7].

Cooperation among wireless SPs, whereby different SPs form a coalition and share their resources, such as spectrum and base station (or access points) and relay nodes, and serve each other other's customers, has the potential to substantially improve the utilization of the available resources. There are a number of recent publications that address cooperation among heterogeneous networks [1]-[7].

In [1], a novel interrelated market model for hierarchical spectrum sharing among primary, secondary, tertiary, and quaternary services is proposed. In [2], the authors propose a simple QoS-based dynamic pricing approach for services provisioning in order to maximize the revenue of SPs and increase user satisfaction level by applying dynamic pricing strategies based on the QoS. In [3], the author investigates heterogeneous network architectures to estimate the pricing for wireless data services. In [4], S. H. Chun etc. study the problem of designing a secondary spectrum-trading market with multiple sellers and multiple buyers and propose a general framework for the trading market based on an auction mechanism. In [5], D. Niyato etc. investigate three different pricing models, namely, market-equilibrium, competitive, and 
cooperative pricing models for spectrum trading in a cognitive radio environment. In [6], $\mathrm{H}$. Chang etc. propose a cooperative spectrum sharing scheme for heterogeneous wireless networks by using a market model, where the equilibrium at which all SPs and all licensed users satisfy the amount of the allocated bandwidth and the price simultaneously is obtained by using the concept of demand and supply from economics. In [7], C.Singh etc. investigate cooperation among providers in wireless networks. The cooperation is modeled by using the theory of transferable payoff coalitional games.

From [1]-[7], It is seen that the heterogeneous networks form a coalition system for maximizing the profit, where they may cooperate and compete. In one hand, it is beneficial for all SPs to cooperatively share spectrum where each SP shares its extra spectrum with other SPs' licensed users to increase the overall network capacity and QoS of the heterogeneous networks. On the other hand, the SPs compete with each other by setting access service prices for attracting more users for more profit. Therefore, in the coalition system, the profit maximization problem becomes more complex due to their interactions. Specifically, the price and QoS strategies of a wireless SP will determine how many customers it can entice (in competition with other SPs) to enhance its profit. Pricing strategy is one of the important factors a SP should consider to maximize its profit. There exists a trade-off between the price and profit: a lower price attracts more customer arrivals while less revenue per customer is achieved; a higher price limits customer arrivals but yields higher per-customer revenue. The user QoS satisfaction also affects the wireless SP's profit, which is described in terms of several factors, such as the probability of blocking and dropping and QoS degradation. Better user QoS enticing more customers can be achieved by occupying more channels, which in turn incurs higher channel leasing cost. Hence, a wireless SP should strike a balance between its tariff and QoS provisioning since better QoS can be achieved by leasing more channels, which in turn incurs higher channel leasing cost.

It is seen that when user requirements (such as the price and QoS) arise, resources of heterogeneous networks related to user requirements are organized. Combination of resources of different networks may lead to many kinds of cooperation modes, while different cooperation modes may result in resource reallocation between networks. Therefore, the user requirements are only the superficies and actually the whole complex coalition system is controlled by the resource allocation modes. Since user requirements vary with time, it is necessary to optimize resource allocation to achieve dynamic balance of each network. Actually, resource optimization process is dynamic evolvement of resource usage from unblance to balance and then from one balance to another balance. Therefore, it is very important for a network to choose adaptive cooperative partners and mechanism to make up for insufficiency of itself limited resource. Therefore, heterogeneous networks can be constructed in a self-organization of structure and reach a certain orderly state.

In this paper we propose to use the basic idea and research results in the field of differential dynamic model [8] to design a general and effective "network cooperation framework" among wireless heterogeneous networks to coordinate the interbehaviors between user and network, network and network, user and user, and also to guide users' consumptions. First, from the point of view of system dynamics, mathematical model is made to study the network resource allocation problem and cooperative and competitive mechanism of heterogeneous networks. Then, structure of the proposed model and its stability are analyzed. And finally system performances are evaluated. Our problem formulation, solution techniques, and results significantly differ from the 
existing literatures. Our focus is on cooperative resource allocation in macroscopic behavior among wireless networks.

The rest of this paper is organized as follows. Section II describes the system model. Section III presents the structure of the cooperative and competitive model and stability analysis. Section IV presents the performance evaluation. Finally, Section V gives the conclusions.

\section{System Model}

We consider a heterogeneous networking scenario, which consists of $N(N \geq 2)$ wireless networks (WNs) belonging to different SPs. As shown in Fig. 1, the SPs form a coalition system for maximizing the profit where they may either compete or cooperate with each other. In the coalition system, the cooperation may include inner-cooperation among users or base stations (or APs) in each network and inter-cooperation between networks. Here our focus is on cooperative resource allocation in macroscopic behavior among wireless networks. Through the cooperation of networks new cooperative effects of the coalition system are produced. Often these effects cannot be even formulated by means of the network alone.

In this paper, the operation state, denoted by $q$, of the coalition system is measured by the network profit. It can be decided by many factors including service tariff, customer satisfaction and QoS provisioning, and coexistence among networks. According to the synergetics theory [9]-[11], although many variables may affect the operation of the coalition system, actually only one or a few variables called order parameters slave the subsystems on the microscopic level. Hence, the proper selection of order parameters appears to be a central issue to model the operation of coalition system. It is found in the presence of collocated SPs, the profit maximization problem becomes more complex due to their interactions. Specifically, the price and QoS strategies of a network drive the customers to choose their service network. Hence, each network will provide reasonable price and guarantee QoS to entice the customers to enhance its profit. As a result, the price and QoS strategies of a network will determine the number of customers choosing different networks, i.e. inducing resource reallocation among networks. Therefore, according to the synergetics theory, we believe the resource allocation mode among the networks is the order parameter determining the macroscopic pattern of the coalition system.

We first assume there is only network $i$ providing the service. And we let $q_{i}(t)$ denote network $i$ 's profit at time $t, q_{i}^{\prime}(t)$ denote profit increasing rate and $f_{i}\left(q_{i}\right)$ denote its instant increment rate (i.e. $\left.q_{i}^{\prime}(t) / q_{i}(t)\right)$. Also we assume the maximum profit of network $i$, denoted by $V_{i}$. According to [8], when $V_{i} \rightarrow \infty$, the network profit will increase with exponent function with fixed instant increment rate $f_{i}\left(q_{i}\right)$, i.e. $q_{i}^{\prime}(t)=r . q_{i}(t)$, where $r$ denotes the profit increment rate in this condition. Actually, $V_{i}$ is always limited. We define $q_{i}(t) / V_{i}$ denoting the profit density. It is easily known the profit increase will become slower and slower with the increase of $q_{i}(t) / V_{i}$. When $q_{i}(t)=V_{i}$, it creases increasing. Here, we use $r \cdot q_{i}(t) / V_{i}$ to express the varying factor. 
Therefore, the profit evolvement of network $i$ can be expressed by

$q_{i}^{\prime}(t)=r \cdot q_{i}(t)\left[\frac{V_{i}-q_{i}(t)}{V_{i}}\right] \equiv q_{i} f\left(q_{i}\right)$

where $\frac{V_{i}-q_{i}(t)}{V_{i}}=1-\frac{q_{i}(t)}{V_{i}}$ denotes trapping (accelerating) effect of the profit increase induced by the increase (decrease) of the profit density. By the first-order conditions, we obtain its equilibrium point as $q_{i}(t)=V_{i}$.

However, when different networks form a coalition system, they may either compete or cooperate with each other. Hence, $f_{i}\left(q_{i}\right)$ is not the only function of $q_{i}$ and it is necessarily related to the state variables of other networks. Therefore, $f_{i}\left(q_{i}\right)$ should be rewritten as $f_{i}(Q)$, where $Q=\left(q_{1}\right.$, $\left.q_{2}, \ldots, q_{n}\right)^{\mathrm{T}}$. Hence, (1) changes into

$\frac{d q_{i}}{d t}=q_{i}(t) f_{i}(Q)$

Cooperation and competition are the inherent attributes of the coalition system. And the interaction between them drives the self-organization evolvement of the system and their interaction degree decides the order and stability of the system. Therefore, we introduce two parameters, $\beta_{i j}\left(-1 \leq \beta_{i j} \leq 1\right)$ and $\delta_{i j}\left(-1 \leq \delta_{i j} \leq 1\right)$, to indicate the competitive and cooperative effect between $\mathrm{WN}_{i}$ and $\mathrm{WN}_{j}$ induced by resource allocation mode among the networks. Since $\mathrm{WN}_{i}$ and $\mathrm{WN}_{j}$ share some common resources, such as spectrum and users, competition for resources occurs between them. Therefore, $\beta_{i j}$ denotes the competitive effect of $\mathrm{WN}_{j}$ taking on $\mathrm{WN}_{i}$. For example, when they completely share common resources, $\beta_{i j}=1$, i.e., $\mathrm{WN}_{j}$ takes a large competitive effect on $\mathrm{WN}_{i}$, and when they share no common resources, we let $\beta_{i j}=0$, i.e., $\mathrm{WN}_{j}$ takes no competitive effect on $\mathrm{WN}_{i}$. And we let $\delta_{i j}$ denote the cooperative effect of $\mathrm{WN}_{j}$ taking on $\mathrm{WN}_{i}$. Similarly, $\delta_{i j}=1$ denotes a large cooperative effect of $\mathrm{WN}_{j}$ taking on $\mathrm{WN}_{i}$ and $\delta_{i j}=0$ indicates no cooperative effect of $\mathrm{WN}_{j}$ taking on $\mathrm{WN}_{i}$. If we assume $f_{i}\left(q_{i}\right)$ is a linear function, then Lokta-Volterra model [12], a differential dynamics model, can be used to model the profit evolvement of network $i$. Therefore, the profit evolvement of the competitive and cooperative networks can be adapted from (2) as

$$
\frac{d q_{i}}{d t}=r_{i} q_{i}\left(1-\frac{q_{i}}{V_{i}}+\sum_{j \neq i} \frac{\delta_{i j} q_{j}}{V_{j}}-\sum_{j \neq i} \frac{\beta_{i j} q_{j}}{V_{j}}\right)
$$


Therefore, the competitive and cooperative mechanism of heterogeneous networks can be modeled by (3). Next we shall see how the interactions of heterogeneous networks give rise to stable structure.

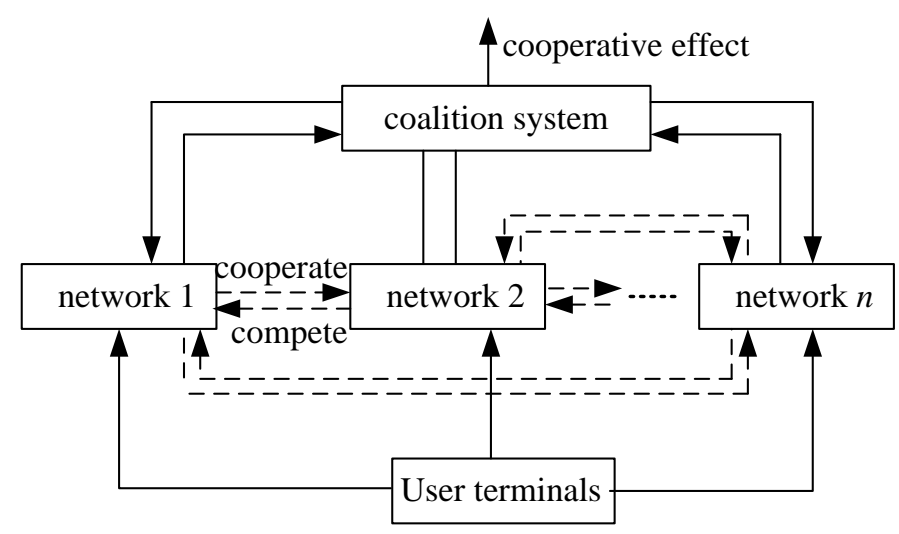

Fig. 1 Coalition system is composed of heterogeneous networks.

\section{Structure of the Cooperative and Competitive Model and Stability Analysis}

In this section, for the sake of clarity of the discussions, we consider two WNs $\left(\mathrm{WN}_{1}\right.$ and $\left.\mathrm{WN}_{2}\right)$. However, the concepts can be easily extended to an arbitrary number of WNs. Then, according to (3), the cooperative and competitive model of two networks can be expressed as follows:

$$
\left\{\begin{array}{l}
\frac{d q_{1}}{d t}=r_{1} q_{1}\left(1-\frac{q_{1}}{V_{1}}+\delta_{12} \frac{q_{2}}{V_{2}}-\beta_{12} \frac{q_{2}}{V_{2}}\right) \equiv q_{1} f_{1}\left(q_{1}, q_{2}\right) \\
\frac{d q_{2}}{d t}=r_{2} q_{2}\left(1-\frac{q_{2}}{V_{2}}+\delta_{21} \frac{q_{1}}{V_{1}}-\beta_{21} \frac{q_{1}}{V_{1}}\right) \equiv q_{2} f_{2}\left(q_{1}, q_{2}\right)
\end{array}\right.
$$

According to the systematic eliminating procedure, the equilibrium points of equation (4) can be obtained by solving

$$
\left\{\begin{array}{l}
r_{1} q_{1}\left(1-\frac{q_{1}}{V_{1}}+\delta_{12} \frac{q_{2}}{V_{2}}-\beta_{12} \frac{q_{2}}{V_{2}}\right)=0 \\
r_{2} q_{2}\left(1-\frac{q_{2}}{V_{2}}+\delta_{21} \frac{q_{1}}{V_{1}}-\beta_{21} \frac{q_{1}}{V_{1}}\right)=0
\end{array}\right.
$$

Hence, four equilibrium points are obtained: $P_{1}(0,0), P_{2}\left(V_{1}, 0\right), P_{3}\left(0, V_{2}\right)$ and

$$
P_{4}\left(\frac{\left[1-\left(\beta_{12}-\delta_{12}\right)\right] V_{1}}{1-\left(\delta_{12}-\beta_{12}\right)\left(\delta_{21}-\beta_{21}\right)}, \frac{\left[1-\left(\beta_{21}-\delta_{21}\right)\right] V_{2}}{1-\left(\delta_{12}-\beta_{12}\right)\left(\delta_{21}-\beta_{21}\right)}\right) .
$$

For any equilibrium point, the corresponding characteristic matrix of evolution equation (5) can be obtained by

$$
\mathbf{A}=\left[\begin{array}{ll}
\partial q_{1} \% \partial q_{1} & \partial q_{1} \% \partial q_{2} \\
\partial q_{2} \% \partial q_{1} & \partial q_{2} \% \partial q_{2}
\end{array}\right]
$$


$\mathbf{A}=\left[\begin{array}{cc}r_{1}\left(1-2 \frac{q_{1}}{V_{1}}+\delta_{12} \frac{q_{2}}{V_{2}}-\beta_{12} \frac{q_{2}}{V_{2}}\right) & r_{1}\left(\delta_{12} \frac{q_{1}}{V_{2}}-\beta_{12} \frac{q_{1}}{V_{2}}\right) \\ r_{2}\left(\delta_{21} \frac{q_{2}}{V_{1}}-\beta_{21} \frac{q_{2}}{V_{1}}\right) & r_{2}\left(1-2 \frac{q_{2}}{V_{2}}+\delta_{21} \frac{q_{1}}{V_{1}}-\beta_{21} \frac{q_{1}}{V_{1}}\right)\end{array}\right]=\left[\begin{array}{cc}l & m \\ n & o\end{array}\right]$.

Then, the characteristic equation of matrix $\mathbf{A}$ is

$\lambda^{2}+b \lambda+c=0$,

where $b=-(l+o)$ and $c=l o-m n$. And, its eigenvalues are

$\lambda_{1}=\frac{-b+\sqrt{b^{2}-4 c}}{2}, \lambda_{2}=\frac{-b-\sqrt{b^{2}-4 c}}{2}$.

According to ordinary differential principle, the stability of equilibrium points can be judged by the plus or minus sign of $b, c, b^{2}-4 c$, or the plus or minus sign of $\lambda_{1}$ and $\lambda_{2}$. Here, we take the former method to judge, i.e., if $c<0$, the equilibrium point is a saddle point; if $b>0, c>0$ and $b^{2}-4 c>0$, the equilibrium point is a stable point; if $b<0, c>0$ and $b^{2}-4 c>0$, the equilibrium point is an unstable point.

Next, we will analyze the stability of competitive and cooperative model.

(1) Input $P_{1}(0,0)$ into $\mathbf{A}$, then $\mathbf{A}$ becomes

$\mathbf{A}_{1}=\left[\begin{array}{ll}r_{1} & 0 \\ 0 & r_{2}\end{array}\right]$

Then, $b=-\left(r_{1}+r_{2}\right)<0, c=r_{1} r_{2}>0$ and $b^{2}-4 c=\left(r_{1}-r_{2}\right)^{2}>0$. Therefore, $P_{1}(0,0)$ is an unstable point. This shows that when the profit of each WN equals to zero, the system is unstable and this case does not exist.

(2) Input $P_{2}\left(V_{1}, 0\right)$ into $\mathbf{A}$, then $\mathbf{A}$ becomes

$$
\mathbf{A}_{2}=\left[\begin{array}{cc}
-r_{1} & r_{1} \frac{V_{1}}{V_{2}}\left(\delta_{12}-\beta_{12}\right) \\
0 & r_{2}\left(1+\delta_{21}-\beta_{21}\right)
\end{array}\right]
$$

Then, $b=r_{1}-r_{2}\left(1+\delta_{21}-\beta_{21}\right)$ and $c=-r_{1} r_{2}\left(1+\delta_{21}-\beta_{21}\right)$. If $1+\delta_{21}-\beta_{21}>0$, then, $c<0$.

Therefore, $P_{2}\left(V_{1}, 0\right)$ is a saddle point. If $1+\delta_{21}-\beta_{21}<0$, then $c>0, b>0$ and $b^{2}-4 c>0$. Therefore, $P_{2}\left(V_{1}, 0\right)$ is a stable point. Since $1+\delta_{21}-\beta_{21}<0$, we can obtain $\delta_{21}<0, \beta_{21}>0$, and simultaneouly $\delta_{21}-\beta_{21}<-1$, which indicates that the competitive negative effect of $\mathrm{WN}_{1}$ taking on $\mathrm{WN}_{2}$ is larger than the cooperative positive effect of $\mathrm{WN}_{1}$ taking on $\mathrm{WN}_{2}$. From the profit of two networks, $\left(V_{1}, 0\right)$, we can see that excessive competition of two 
networks makes no increase of profit for $\mathrm{WN}_{1}$ while the profit of $\mathrm{WN}_{2}$ is 0 .

(3)Input $P_{3}\left(0, V_{2}\right)$ into $\mathbf{A}$, then $\mathbf{A}$ becomes

$\mathbf{A}_{3}=\left[\begin{array}{cc}r_{1}\left(1+\delta_{12}-\beta_{12}\right) & 0 \\ r_{2}\left(\delta_{21} \frac{V_{2}}{V_{1}}-\beta_{21} \frac{V_{2}}{V_{1}}\right) & -r_{2}\end{array}\right]$.

Then, $b=r_{2}-r_{1}\left(1+\delta_{12}-\beta_{12}\right)$ and $c=-r_{1} r_{2}\left(1+\delta_{12}-\beta_{12}\right)$. If $1+\delta_{12}-\beta_{12}>0$, then $c<0$.

Therefore, $P_{3}\left(0, V_{2}\right)$ is a saddle point. If $1+\delta_{12}-\beta_{12}<0$, then $c>0, b>0$ and $b^{2}-4 c>0$. Therefore, $P_{3}\left(0, V_{2}\right)$ is a stable point. Similar to the analysis on $P_{2}\left(V_{1}, 0\right)$, since $1+\delta_{12}-\beta_{12}<0$, we can obtain $\delta_{12}<0, \beta_{12}>0$, and simultaneouly $\delta_{12}-\beta_{12}<-1$, which indicates that the competitive effect of $\mathrm{WN}_{2}$ taking on $\mathrm{WN}_{1}$ is more than the cooperative effect of $\mathrm{WN}_{2}$ taking on $\mathrm{WN}_{1}$. From the profit of two SPs, $\left(0, V_{2}\right)$, we can see that excessive competition of two SPs makes no increase of profit of $\mathrm{WN}_{2}$ while the profit of $\mathrm{WN}_{1}$ is 0 .

(4)Input $P_{4}\left(\frac{\left[1-\left(\beta_{12}-\delta_{12}\right)\right] V_{1}}{1-\left(\delta_{12}-\beta_{12}\right)\left(\delta_{21}-\beta_{21}\right)}, \frac{\left[1-\left(\beta_{21}-\delta_{21}\right)\right] V_{2}}{1-\left(\delta_{12}-\beta_{12}\right)\left(\delta_{21}-\beta_{21}\right)}\right)$ into $\mathbf{A}$, then $\mathbf{A}$ becomes $\mathbf{A}_{4}=\left[\begin{array}{ll}E & F \\ G & H\end{array}\right]$,

where $E=\frac{-r_{1}\left(1+\delta_{12}-\beta_{12}\right)}{1-\left(\delta_{12}-\beta_{12}\right)\left(\delta_{21}-\beta_{21}\right)}, \quad F=\frac{r_{1} V_{1}}{V_{2}} \times \frac{\left(\delta_{12}-\beta_{12}\right)\left[1+\left(\delta_{12}-\beta_{12}\right)\right]}{1-\left(\delta_{12}-\beta_{12}\right)\left(\delta_{21}-\beta_{21}\right)}$, $G=\frac{r_{2} V_{2}}{V_{1}} \times \frac{\left(\delta_{21}-\beta_{21}\right)\left[1-\left(\delta_{21}-\beta_{21}\right)\right]}{1-\left(\delta_{12}-\beta_{12}\right)\left(\delta_{21}-\beta_{21}\right)}$, and $H=\frac{-r_{2}\left(1+\delta_{21}-\beta_{21}\right)}{1-\left(\delta_{12}-\beta_{12}\right)\left(\delta_{21}-\beta_{21}\right)}$

Then, we obtain $b=\frac{r_{1}\left(1+\delta_{12}-\beta_{12}\right)+r_{2}\left(1+\delta_{21}-\beta_{21}\right)}{1-\left(\delta_{12}-\beta_{12}\right)\left(\delta_{21}-\beta_{21}\right)}, c=\frac{r_{1} r_{2}\left[1+\delta_{12}-\beta_{12}\right)\left(1+\delta_{21}-\beta_{21}\right)}{1-\left(\delta_{12}-\beta_{12}\right)\left(\delta_{21}-\beta_{21}\right)}$. and $b^{2}-4 c=\left[\frac{r_{1}\left(1+\delta_{12}-\beta_{12}\right)-r_{2}\left(1+\delta_{21}-\beta_{21}\right)}{1-\left(\delta_{12}-\beta_{12}\right)\left(\delta_{21}-\beta_{21}\right)}\right]^{2} \geq 0$.

In this case, in order to judge the plus or minus sign of $b, c, b^{2}-4 c$, the following four conditions need to be discussed.

(i) When $1+\delta_{12}-\beta_{12}>0$ and $1+\delta_{21}-\beta_{21}>0$, then $1-\left(\delta_{12}-\beta_{12}\right)\left(\delta_{21}-\beta_{21}\right)>0$. Thus in this way, $c>0, b>0$ and $b^{2}-4 c>0$. Therefore, $P_{4}$ is a stable point. That is to say, the coalition system is in stable state when $\delta_{i j}>0$, or $\beta_{i j}<0$, and or $\delta_{i j}<0, \beta_{i j}>0$ and simultaneously 
$\delta_{i j}-\beta_{i j}>-1$. This shows that the conditions that the coalition system is stable are that the cooperative effect on the partner network is bigger than the competitive effect on the partner network or the competitive effect is so small that it cannot induce the coalition system fluctuation. In this case, the profit of two networks is respectively given by

$$
q_{1}=\frac{\left[1-\left(\beta_{12}-\delta_{12}\right)\right] V_{1}}{1-\left(\delta_{12}-\beta_{12}\right)\left(\delta_{21}-\beta_{21}\right)}
$$

and

$$
q_{2}=\frac{\left[1-\left(\beta_{21}-\delta_{21}\right)\right] V_{2}}{1-\left(\delta_{12}-\beta_{12}\right)\left(\delta_{21}-\beta_{21}\right)}
$$

Since,

$$
q_{1}>V_{1} \text { and } q_{2}>V_{2} \text {, then } q_{1}+q_{2}>V_{1}+V_{2} \text {. }
$$

Therefore, under the stable state of $P_{4}$, the profit produced by the two networks with cooperation is bigger than that of the two networks without cooperation.

(ii) When $1+\delta_{12}-\beta_{12}<0$ and $1+\delta_{21}-\beta_{21}>0$, if $\left[1-\left(\delta_{12}-\beta_{12}\right)\left(\delta_{21}-\beta_{21}\right)\right]>0$, then $\mathrm{b}<0$.

Therefore, $P_{4}$ is a saddle point. It shows the competitive effect of the coalition system is larger than the cooperative effect. In this case the coalition system is unstable.

If $\left[1-\left(\delta_{12}-\beta_{12}\right)\left(\delta_{21}-\beta_{21}\right)\right]<0$ and $-r_{1}\left(1+\delta_{12}-\beta_{12}\right)>r_{2}\left(1+\delta_{21}-\beta_{21}\right)$, then $b>0$ and $a>0$. It is still a stable point. It shows that the competitive negative effect of $\mathrm{WN}_{1}$ taking on $\mathrm{WN}_{2}$ is larger than the cooperative positive effect of $\mathrm{WN}_{1}$ taking on $\mathrm{WN}_{2}$ while the effect of $\mathrm{WN}_{1}$ taking on $\mathrm{WN}_{2}$ is bigger. But when $-r_{1}\left(1+\delta_{12}-\beta_{12}\right)<r_{2}\left(1+\delta_{21}-\beta_{21}\right), a<0$. Therefore, it is unstable. It shows the excessive competition will disintegrate the system.

When $1+\delta_{12}-\beta_{12}>0$ and $1+\delta_{21}-\beta_{21}<0$, analysis are similar.

(iii) When $1+\delta_{12}-\beta_{12}<0$ and $1+\delta_{21}-\beta_{21}<0$, then $1-\left(\delta_{12}-\beta_{12}\right)\left(\delta_{21}-\beta_{21}\right)<0$. Hence, $b<0$. Therefore, it is a saddle point. It shows the excessive competition will make the system unstable and finally disintegrate the system.

\section{Performance Evaluation}

We evaluate the performance of our proposed model by Vensim [12] which is used for developing, analyzing, and packaging dynamic feedback models. We consider a coalition system consisting of $\mathrm{WN}_{1}$ and $\mathrm{WN}_{2}$. The program by DYNAMO language for the cooperative and competitive model of $\mathrm{WN}_{1}$ and $\mathrm{WN}_{2}$ is shown in Fig. 2. According to the program, the corresponding system dynamics model is shown in Fig. 3. 


$$
\begin{array}{ll}
L & q 1 . K=q 1 . J+D T \cdot(I R 1 . J K-O R 1 . J K) \\
R & I R 1 . K L=R 11 . K+R 12 . K \\
A & R 11 . K=r 1 * q 1 . K \\
A & R 12 . K=d 12^{*} r 1^{*} q 1 . K * q 2 . K / V 2 \\
R & O R 1 . K L=R 13 . K+R 14 . K \\
A & R 13 . K=r 1 * q 1 . K * q 1 . K / V 1 \\
A & R 14 . K=b 12 * r 1 * q 1 . K * q 2 . K / V 2 \\
L & q 2 . K=q 2 . J+D T \cdot(I R 2 . J K-O R 2 . J K) \\
R & I R 2 . K L=R 21 . K+R 22 . K \\
A & R 21 . K=r 2 * q 2 . K \\
A & R 22 . K=d 21 * r 2 * q 1 . K * q 2 . K / V 1 \\
R & O R 2 . K L=R 23 . K+R 24 . K \\
A & R 23 . K=r 2 * q 2 . K * q 2 . K / V 2 \\
A & R 24 . K=b 21 * r 2 * q 1 . K * q 2 . K / V 1
\end{array}
$$

Fig. 2 Program by DYNAMO language for the cooperative and competitive model of $\mathrm{WN}_{1}$ and $\mathrm{WN}_{2}$

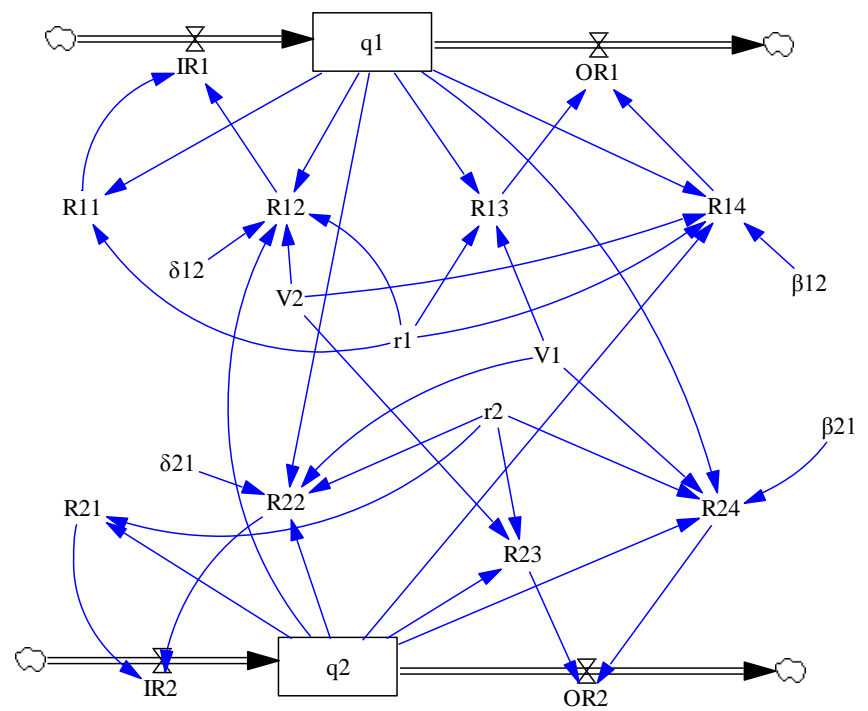

Fig. 3 System dynamics model of two networks with cooperation and competition

In this set of experiments, we assume the maximum profits of $\mathrm{WN}_{1}$ and $\mathrm{WN}_{2}$ are 150 and 100 respectively in non-cooperative scheme where each SP serves its LUs with all of its bandwidth respectively, i.e., $V_{1}=150$ and $V_{2}=100$. Fig. 4 shows the profit evolving curves with time for $\mathrm{WN}_{1}$ and $\mathrm{WN}_{2}$ with different natural growth rates (i.e., $r_{1}$ and $r_{2}$ ) under the conditions of non-cooperation and non-competition, i.e., $\delta_{12}=\beta_{12}=\delta_{21}=\beta_{21}=0$. We observe that the large natural growth rate cannot increase the profit of the network but can make the network use less time to achieve the equilibrium point, i.e. $(150,100)$.

Fig. 5 shows the profit evolving curves for $\mathrm{WN}_{1}$ and $\mathrm{WN}_{2}$ with time, where $V_{1}=150, V_{2}=100$, $r_{1}=r_{2}=0.01, \delta_{12}=0.5, \delta_{21}=0.6, \beta_{12}=0.3, \beta_{21}=0.4$. We compare the profit of each SP in 
cooperative spectrum sharing scheme (denoted by $\mathrm{CWN}_{1}$ and $\mathrm{CWN}_{2}$ ) with the maximum profit of each SP in non-cooperative scheme (denoted by $\mathrm{WN}_{1}$ and $\mathrm{WN}_{2}$ ). It is seen from the figure that when the cooperative coefficient is larger than the competitive coefficient, the system can reach equilibrium point $(187.49,125.00)$ and it is also a stable equilibrium point. In this case, the increased percentages of each SP's profit by this cooperative spectrum sharing scheme are $24.99 \%$ and 25\% respectively. Therefore, all SPs gain extra incentives. It is also seen that only if the heterogeneous system evolves with cooperation, can the coalition system reach the stable state with time going on.

Fig. 6 shows the profit evolving curves for $\mathrm{WN}_{1}$ and $\mathrm{WN}_{2}$ when the cooperative coefficient is less than the competitive coefficient. It is seen that in this case each SP's profit will decrease. Therefore, the excessive competition will decrease each SP's profit and make the system unstable.

Fig.7 shows the profit evolving curves for $\mathrm{WN}_{1}$ and $\mathrm{WN}_{2}$ with time, where $V_{1}=V_{2}=100$, $r_{1}=r_{2}=0.05, \delta_{12}=0.1, \delta_{21}=0.1, \beta_{12}=0.8, \beta_{21}=0.7$. In this set of experiments, the two networks have the same network scale and natural growth rate, but the cooperative coefficient is less than the competitive coefficient. It is also seen from the figure that with time going on, the profit of one network will decrease and the profit of the other network will increase. As a result, the coalition system will not be stable and finally disintegrated. Therefore, severe competition may result in lower price and may shrink total profit of SPs in turn. As an extreme result, one SP cannot increase the profit due to lower price although it can attract all the users, and the other SP has no users and so there is no profit at all.
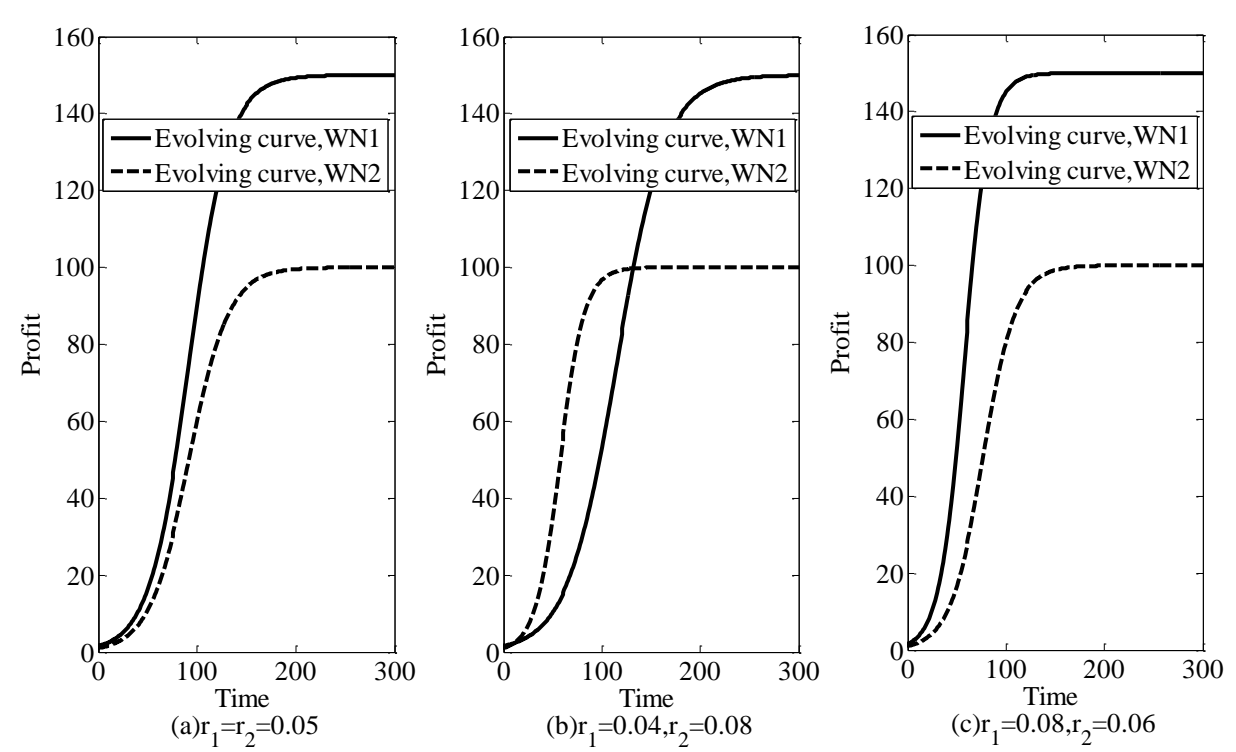

Fig. 4 Profit evolving curves with time, where $V_{1}=150, V_{2}=100$, and $\delta_{12}=\beta_{12}=\delta_{21}=\beta_{21}=0$. 


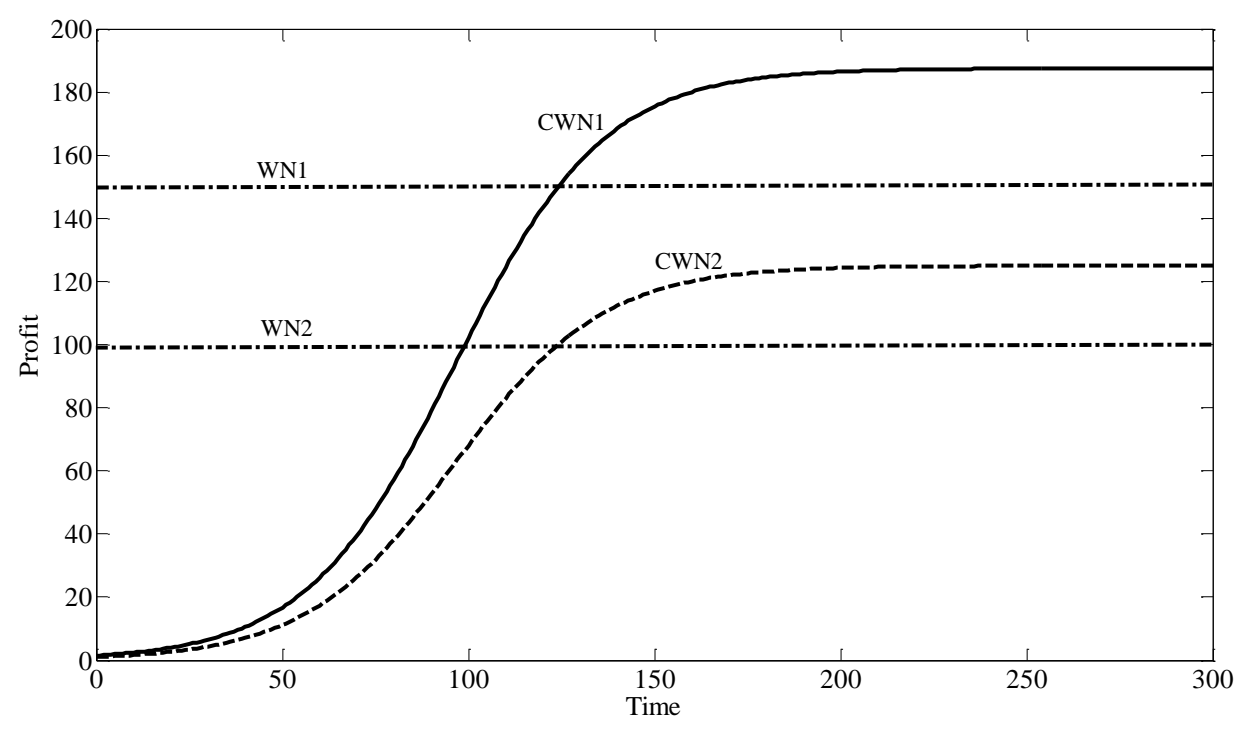

Fig. 5 Profit evolving curves with time, where $V_{1}=150, V_{2}=100, r_{1}=r_{2}=0.01, \delta_{12}=0.5$, $\delta_{21}=0.6, \beta_{12}=0.3, \beta_{21}=0.4$.

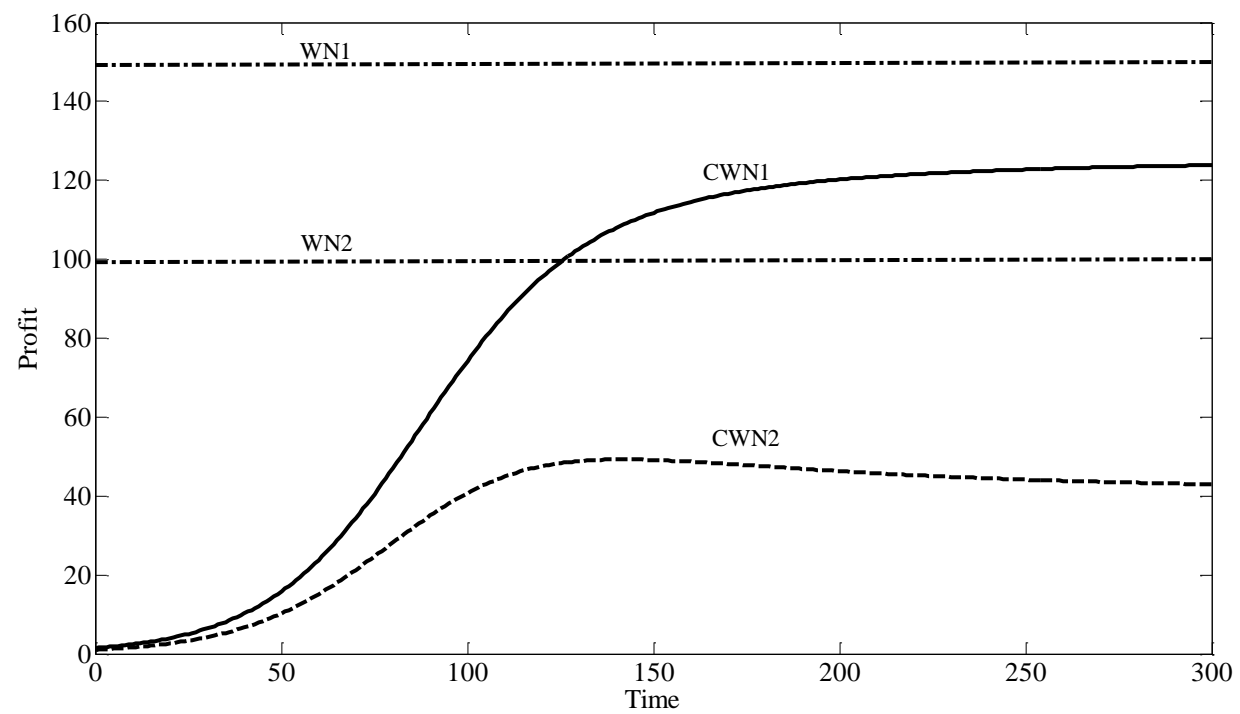

Fig. 6 Profit evolving curves with time, where $V_{1}=150, V_{2}=100, r_{1}=r_{2}=0.05, \delta_{12}=0.1$, $\delta_{21}=0.2, \beta_{12}=0.5, \beta_{21}=0.9$. 


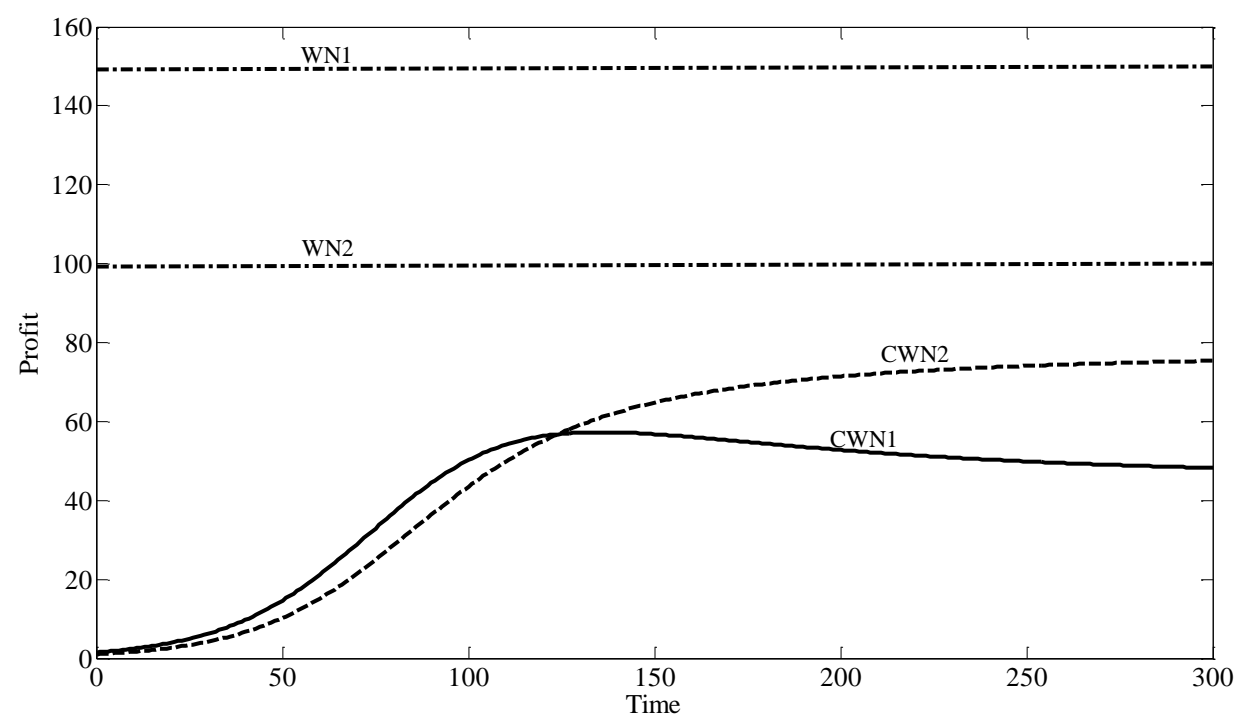

Fig. 7 Profit evolving curves with time, where $V_{1}=V_{2}=100, r_{1}=r_{2}=0.05, \delta_{12}=0.1$,

$$
\delta_{21}=0.1, \beta_{12}=0.8, \beta_{21}=0.7
$$

\section{Conclusions}

The heterogeneous networks form a coalition system for maximizing the profit, where they may either compete or cooperate with each other. In this paper, we introduce Lokta-Volterra model, to build SPs' profit evolvement model which considers natural growth rate of the network itself and cooperative and competitive effects among networks. Then, structure of the proposed cooperative and competitive model and its stability analysis are given. And system performances are evaluated by Vensim. Analysis and simulation results show that the stability of the coalition system depends on the cooperative mechanism of its subsystems. When the coalition system approaches the evolving stable state, the profit of each SP in cooperative scheme is larger than that of each SP in non-cooperative scheme. Therefore, the cooperation of the coalition system optimizes the system architecture and increases the total profit.

\section{REFERENCES}

[1] D. Niyato and E. Hossain, "A microeconomic model for hierarchical bandwidth sharing in dynamic spectrum access networks," IEEE Transactions on Computers, vol. 59, no. 7, pp. 865 -877, July 2010.

[2] A. Guerrero-Iban andez, C. Flores-Corte ands, A. Barba, and A. Reyes, “A quality of service-enabled pricing approach for heterogeneous wireless access networks," in Intelligent Environments (IE), 2010 Sixth International Conference on, July 2010, pp. $231-236$

[3] D. Krishnaswamy, "Network economics considerations for incremental data services in heterogeneous wireless wide area networks," in Vehicular Technology Conference Fall (VTC 2010-Fall), 2010 IEEE 72nd, Sept. 2010, pp. 1 -5.

[4] Sung Hyun Chun and Richard J. La, "Secondary Spectrum Trading-Auction-Based Framework for Spectrum Allocation and Profit Sharing,” IEEE/ACM TRANSACTIONS ON NETWORKING, accepted.

[5] D. Niyato and E. Hossain, "Market-Equilibrium, Competitive, and Cooperative Pricing for Spectrum Sharing in Cognitive Radio Networks: Analysis and Comparison,” IEEE Trans. Wireless Commun., vol. 7, no. 11, Nov. 2008. 
[6] Hung-Bin Chang and Kwang-Cheng Chen, "Cooperative Spectrum Sharing Economy for Heterogeneous Wireless Networks,” in IEEE GLOBECOM Workshops, pp 458-463, Dec. 2011.

[7] C.Singh, S.Sarkar, A.Aram, and A.Kumar, "Cooperative Profit Sharing in Coalition-Based Resource Allocation in Wireless Networks,” IEEE/ACM TRANSACTIONS ON NETWORKING, VOL. 20, NO. 1, FEBRUARY 2012.

[8]David H. Romer, 2000. "Keynesian Macroeconomics without the LM Curve," Journal of Economic Perspectives, American Economic Association, vol. 14(2),pp. 149-169.

[9] Haken, H. (ed.): Synergetics. Cooperative Effects in Multicomponent Systems. Stuttgart: Teubner 1973.

[10] M. Bushev, Synergetics: Chaos, Order, Self-Organization. Singapore: World Scientific, 1994.

[11] Haken, H. Information and Self-Organization, A Macroscopic Approach to Complex Systems; Springer: New York, NY, 2006.

[12] http://vensim.com/vensim-software/. 